\title{
STRONG OSCILLATIONS FOR SECOND ORDER NONLINEAR FUNCTIONAL DIFFERENTIAL EQUATIONS
}

\author{
JURANG YAN \\ Department of Mathematics \\ Shanxi University \\ Talyuan, Shanx 1 \\ People's Repub1ic of China \\ (Received Ju1y 28, 1986 and in revised form May 5, 1987)
}

ABSTRACT. In this paper, we establish some strongly oscillation theorems for nonlinear second order functional differential equation

$$
x^{\prime \prime}(t)+p(t) f(x(t), x(g(t)))=0
$$

without assuming that $g(t)$ is retarded or advanced.

KEY WORDS AND PHRASES. Strong oscillation, nonlinear, functional differential equations.

1980 AMS SUBJECT CLASSIFICATION CODE. Primary $34 \mathrm{~K} 15$.

1. INTRODUCTION. We consider the second order nonlinear functional differential equation

$$
x^{\prime \prime}(t)+p(t) f(x(t), x(g(t)))=0
$$

where $p(t), g(t) \varepsilon C\left(\left[t_{0}, \infty\right), R\right), g(t)+\infty$ as $t+\infty$ and $f(u, v) \varepsilon c(R, R)$ and has the sign of $u$ and $v$ when they have the same sign. We shall restrict our attention to solutions of (1.1) which exist on some positive half-1ine. A nontrivial solution $x(t)$ is called oscillatory if $x(t)$ has an unbounded set of zeros, and otherwise it is called nonoscillatory. Equation (1.1) is said to be oscillatory if every solution of (1.1) is oscillatory.

Oscillation theory for (1.1) has been developed by many authors. Bradley [1], Chiou [2], Erbe [3] Gollwitzer [4], Ladas [5], Travis [6], Waltman [7], Wong [8] and references therein. It is wellknown theorem of Wintner [9] and Leighton [10] that the linear equation

$$
x^{\prime \prime}(t)+p(t) x(t)=0
$$

is oscillatory if $\int^{\infty} p(t) d t=\infty$, even $p(t)$ is not assumed nonnegative. Bradley [1] 
and Waltman [7] demonstrated that the equation

$$
x^{\prime \prime}(t)+p(t) x(g(t))=0
$$

is oscillatory if $p(t)>0$ and $\int^{\infty} p(t) d t=\infty$. Travis [6] constructed a counterexample showing that the Leighton-Wintner oscillation theorem can not be extended to Equation (1.2) unless $p(t)>0$. The author [11] extended Bradley-Waltman oscillation theorem to (1.1) i.e. if $p(t)>0$ and $\int^{\infty} p(t) d t=\infty$, then $(1.1)$ is ocillatory.

The purpose of this paper is to establish some strongly oscillation criteria for (1.1). We are primarily interested in the case when $p(t)>0, \int^{\infty} p(t) d t<\infty$, are satisfied.

Considering the equation

$$
x^{\prime \prime}(t)+\lambda p(t) x(t)=0,
$$

We shall call $p(t)$ a strongly oscillatory coefficlent if (1.3) is osc1llatory for all positive $\lambda$. If $p(t)>0$, Nehari [12] shows that

$$
\lim _{t \rightarrow \infty} \sup t \int_{t}^{\infty} p(s) d s=\infty
$$

is a necessary and sufficient condition for $p(t)$ to be a strongly osclllatory coefficient. In general, motivated by Nehari, we define as follows: Fquation (1.1) is said to be strongly oscillatory if the related equation of $(1.1)$

$$
x^{\prime \prime}(t)+\lambda p(t) f(x(t), x(g(t)))=0
$$

is osillatory for all positive $\lambda$.

\section{MAIN RESULTS.}

For Equation (1.1) the following conditions are assumed to hold throughout the paper:

(i) $p(t)>0$ and there exists $h(t)<\min (g(t), t)$ such that $0<k<h^{\prime}(t)$ where k is a constant.

(ii) there exists $m>0$ such that $|u|>m$ implies

$$
\lim _{|v| \rightarrow \infty} \frac{|f(u, v)|}{|\phi(v)|} \geqslant \varepsilon_{1}>0
$$
where $\phi(v) \in C^{\prime}(R), v \phi(v)>0$ and $\phi^{\prime}(v) \neq 0$ for $v \neq 0$, and $|\lim |+\infty \phi^{\prime}(v)>\delta>0$
where $\Sigma_{1}$ and $\delta$ are constants.

We begin with a Lemma which needed in establishing our results. 
LEMMA 2.1. Suppose that for $\lambda=\lambda_{0}>0$ Equation (1.1) has a nonoscillatory solution $x(t)$. Then the following inequality holds for all large $t$,

$$
w(t)>\sigma \int_{t}^{\infty} w^{2}(s) d s+\lambda_{0} \varepsilon \int_{t}^{\infty} p(s) d s,
$$

where $w(t)=x^{\prime}(t) / \phi(x(h(t))), \sigma$ and $\varepsilon$ are positive constants.

PROOF. Assume that Equation (1.4) at $\lambda=\lambda_{0}$, has a nonoscillatory solution $x(t)>0$ for $t>t_{0}>0$. A similar proof will hold if $x(t)<0$ for $t>t_{0}$. It is easy to verify that $x^{\prime \prime}(t)<0$ and $x^{\prime}(t)>0$ for all large $t$. Let $w(t)=x^{\prime}(t) / \phi(x(h(t)))$, then

$$
w^{\prime}(t)=-\lambda_{0} p(t) \frac{f(x(t), x(g(t)))}{\phi(x(h(t)))}-\frac{\phi^{\prime}(x(h(t))) x^{\prime}(h(t)) h^{\prime}(t)}{\phi(x(h(f)))} w(t) .
$$

Since $x^{\prime}(t)>0$ for large $t, \lim _{t \rightarrow \infty} x(t)$ exists either as a finite or infinite 1 imit. If $\lim _{t \rightarrow \infty} x(t)=\alpha$ is finite, then

$$
\lim _{t \rightarrow \infty} \frac{f(x(t), x(g(t)))}{\phi(x(g(t)))}=\frac{f(\alpha, \alpha)}{\phi(\alpha)}=\varepsilon_{2}>0 .
$$

If $\lim _{t \rightarrow \infty} x(t)=\infty$, then by (ii) we have that

$$
\frac{f(x(t), x(g(t)))}{\phi(x(g(t)))}>\varepsilon_{1}
$$

for large $t$. In efther case, for sufficiently large $t$, we have that

$$
\frac{f(x(t), x(g(t)))}{\phi(x(g(t)))}>\varepsilon \text {, where } \varepsilon=\min \left(\varepsilon_{1}, \varepsilon_{2}\right)
$$

Since $x(t)$ is increasing, for large $t$ we have that

$$
p(t) \frac{f(x(t), x(g(t)))}{\phi(x(h(t)))}>p(t) \frac{f(x(t), x(g(t)))}{\phi(x(g(t)))}>\lambda_{0} \varepsilon_{p}(t)
$$

and in view of $x^{\prime \prime}(t)<0$ for large $t$ and $(i 1)$ we see that

$$
\frac{\phi^{\prime}(x(h(t))) x^{\prime}(h(t)) h^{\prime}(t)}{\phi(x(h(t)))} w(t) \geqslant \frac{\phi^{\prime}(x(h(t))) x^{\prime}(t) h^{\prime}(t)}{\phi(x(h(t)))} \geqslant k \delta w^{2}(t)=\sigma w^{2}(t)
$$

Thus for $t>t_{1}>t_{0}$,

and

$$
w^{\prime}(t)+\sigma w^{2}(t)+\lambda_{0} \varepsilon p(t)<0
$$

$$
w^{\prime}(t)+\sigma w^{2}(t)<0 .
$$

From (2.4), we have that

$$
\frac{d}{d t}\left(-\frac{1}{w(t)}+\sigma t\right)<0 .
$$


Integrating the above inequality from $t_{1}$ to $t$ we obtain that

$$
0<w(t)<\frac{1}{\sigma t+b}
$$

where $b=\frac{1}{w\left(t_{1}\right)}-k t_{1}$. From $(2.5)$ we have that

$$
\lim _{t \rightarrow \infty} w(t)=0
$$

Integrating (2.3) from $t_{1}$ to $t$, then letting $t \rightarrow \infty$ we obtain that (2.1) holds for all large.

We introduce the function sequence $\left\{p_{n}(t)\right\}, n=0,1,2, \ldots$, defined as follows:

$$
\begin{aligned}
& p_{0}(t)=p(t), \quad p_{1}(t)=\int_{t}^{\infty} p_{0}(s) d s, \\
& p_{n+1}(t)=\int_{t}^{\infty} p_{n}^{2}(s) d s, \quad n=1,2,3, \ldots .
\end{aligned}
$$

THEOREM 2.2. As sume that one of the following conditions holds, $\left(I_{1}\right)$ there is an integer $m>1$ such that $p_{n}(t)$ is defined for $n=1,2, \ldots, m$, and

$$
\lim _{t \rightarrow \infty} \sup \operatorname{tp}_{m}(t)=\infty
$$

$\left(I_{2}\right)$ there is an integer $m \geqslant 2$ such that $p_{n}(t)$ is defined for $n=1,2, \ldots, m-1$, but $p_{m}(t)$ does not exist, i.e.

$$
\int^{\infty} \mathrm{p}_{\mathrm{m}-1}^{2}(t) \mathrm{dt}=\infty
$$

Then equation (1.1) is strongly oscillatory.

PROOF. Assume to the contrary that Equation (1.4) at $\lambda=\lambda_{0}>0$, has a nonoscillatory solution $x(t)>0$ for $t>t_{0}>0$. A similar argument holds when $x(t)<0$ for $t>t_{0}>0$. Let $w(t)=x^{\prime}(t) / \phi(x(h(t)))$. As in the proof of Lemma 2.1 , we can obtain

$$
w^{\prime}(t)+\sigma w^{2}(t)+\lambda_{0} \varepsilon_{p_{0}}(t)<0, t>t_{1}>t_{0}
$$

Suppose $m=1$. Define $u_{0}(t)=\sigma w(t)$; then

$$
u_{0}^{\prime}(t)+u_{0}^{2}(t)+\xi_{0} p_{0}(t)<0, t>t_{1}>t_{0}
$$

where $\xi_{0}=\lambda_{0} \varepsilon \sigma$. However, by a we11-known theorem of Wintner [13] this implies the equation

$$
y^{\prime \prime}(t)+\xi_{0} p_{0}(t) y(t)=0
$$

is nonoscillatory. This contradicts the fact that the condition ( $I_{1}$ ) implies $\mathrm{p}_{0}(t)$ is a strongly oscillatory coefficient.

If $m>1$, integrating (2.10) from $t_{1}$ to $t$ we obtain 


$$
u_{0}(t)-u\left(t_{1}\right)+\int_{t_{1}}^{t} u_{0}^{2}(s) d s+\xi_{0} \int_{t_{1}}^{t} p_{0}(s) d s<0 .
$$

Since $\lim _{t \rightarrow \infty} u_{0}(t)=\lim _{t \rightarrow \infty} \sigma w(t)=0$, from (2.12) we get

$$
u_{0}(t)>u_{1}(t)+\xi_{0} p_{1}(t), \quad t>t_{1},
$$

where $u_{1}(t)=\int_{t}^{\infty} u_{0}^{2}(s) d s$. Then (2.13) implies that

$$
u_{1}^{\prime}(t)=-u_{0}^{2}(t)<-u_{1}^{2}(t)-2 \xi_{0} u_{1}(t) p_{1}(t)-\xi_{0}^{2} p_{1}^{2}(t) \text {. }
$$

Hence

$$
u_{1}^{\prime}(t)+u_{1}^{2}(t)+\xi_{1} p_{1}^{2}(t) \leqslant 0
$$

where $\xi_{1}=\xi_{0}^{2}$.

If $m=2$, then by the Nehari theorem, the condition $\left(I_{1}\right)$ implies equation

$$
y^{\prime \prime}(t)+\xi_{1} p_{1}^{2}(t) y(t)=0
$$

is oscillatory, contradicting (2.14).

If the condition $\left(I_{2}\right)$ is satisfied, then by the Leighton-Wintner theorem we have that equation (2.15) is oscillatory, again a contradiction.

When $m>2$, we obtain inductively that

$$
u_{m-1}^{\prime}(t)+u_{m-1}^{2}(t)+\xi_{m-1} p_{m-1}^{2}(t)<0, \quad t>t^{\star}>t_{1} \cdot
$$

where $u_{m-1}(t)=\int_{t}^{\infty} u_{m-2}^{2}(s) d s, \xi_{m-1}$ is a constant and $p_{m-1}(t)$ is

defined by (2.7). Applying the Wintner theorem again, it follows that equation

$$
y^{\prime \prime}(t)+\xi_{m-1} p_{m-1}^{2}(t) y(t)=0
$$

is nonoscillatory. But this contradicts again the fact that the condition $\left(I_{1}\right)$ or $\left(I_{2}\right)$ implies that equation (2.17) is oscillatory. The proof is thus complete.

REMARK. Theorem 2.2 includes Theorem 2.2 in [6] as a special case, i.e. $\phi(u)=u$ and $m=1$.

Consider the function sequence $\left.\operatorname{lq}_{n}(t, \xi, n)\right\}, n=1,2, \ldots$, which is defined as follows:

$$
\begin{aligned}
& q_{0}(t, n)=n \int_{t}^{\infty} p(s) d s, q_{1}(t, \xi, n)=\xi \int_{t}^{\infty} q_{0}^{2}(s, n) d s+q_{0}(t, n), \\
& q_{n}(t, \xi, n)=\xi \int_{t}^{\infty} q_{n-1}^{2}(s, \xi, n) d s+q_{0}(t, n), \quad t>t_{0}, n=2,3, \ldots .
\end{aligned}
$$

where $\zeta$ and $n$ are positive constants. 
THEOREM 2.3. For all positive constants $\xi$ and $n$ assume that any one of following conditions is satisfied:

(II $)$ there is a positive integer m such that $q_{n}(t, \xi, n)$ is defined for $n=1,2, \ldots, m-1$, but $q_{m}(t, \xi, n)$ does not exist;

$\left(I_{2}\right) \quad q_{n}(t, \xi, n)$ is defined for $n=1,2, \ldots$, but the function sequence $(2.18)$ is not convergent for all large $t$.

$\left(I_{3}\right)$ the sequence $(2.18)$ is convergent and 1 im $q_{n}(t, \xi, \eta)=q(t, \xi, \eta)$, but $q(t, \xi, n) \notin L^{2}(t, \infty)$.

Then equation (1.4) is strongly oscillatory.

PRO0F. Assume that Equation (1.1) at $\lambda=\lambda_{0}>0$, has a solution $x(t)>0$ for $t>t_{0}>0$. A similar proof will hold if $x(t)<0$ for $t>t_{0}$. Let $w(t)=x^{\prime}(t) / \phi(x(h(t)))$. From Lemma 2.1 we can obtain (2.1). It follows that $w(t)>q_{0}\left(t, n_{0}\right)$, where $\eta_{0}=\lambda_{0} \varepsilon_{\text {. Hence }}$

$$
w^{2}(t)>q_{0}^{2}\left(t, n_{0}\right), \quad t>t_{1}>t_{0}
$$

Suppose that (II ) holds. If $m=1$ from (2.1), (2.19) Implies that

$$
\begin{aligned}
& \int_{t}^{\infty} q_{0}^{2}\left(s, n_{0}\right) d s<\int_{t}^{\infty} w^{2}(s) d s<\infty \text {. Thus } \\
& \quad q_{1}\left(t, \xi_{0}, \eta_{0}\right)=\xi_{0} \int_{t}^{\infty} q_{0}^{2}\left(s, \eta_{0}\right) d s+q_{0}\left(t, \eta_{0}\right)<w(t), t>t_{1}
\end{aligned}
$$

where $\xi_{0}=\sigma$. This is in contradiciton to the nonexistence of $q_{1}$. If $m>1$, from $(2.1)$ and (2.19) we get that $q_{m-1}<w(t)$. Hence $\int_{t}^{\infty} q_{m-1}\left(s, \xi_{0} n_{0}\right) d s<\int_{t}^{\infty} w^{2}(s) d s<\infty$. Applying $(2.1)$ we have that

$$
q_{m}\left(t, \xi_{0}, n_{0}\right)=\xi \int_{t}^{\infty} q_{m-1}^{2}\left(s, \xi_{0}, n_{0}\right) d s+q_{0}\left(t, n_{0}\right)<w(t)
$$

and we arrive at a contradiction of $\left(\mathrm{II}_{1}\right)$.

Suppose that $\left(\mathrm{II}_{2}\right)$ holds. From (2.18) and (2.19), we conclude that for all large $t$

$$
q_{n-1}\left(t, \xi_{0}, n_{0}\right)<q_{n}\left(t, \xi_{0}, n_{0}\right)<w(t), n=1,2, \ldots \ldots
$$

Therefore $\lim _{n \rightarrow \infty} q_{n}\left(t, \xi_{0}, n_{0}\right)$ exists and has a finte limit. But this constradicts the fact that $q_{n}\left(t, \xi_{\delta} n_{0}\right)$ is not convergent.

Suppose that $\left(\mathrm{II}_{3}\right)$ holds. By $(2.20)$,

$$
\lim q_{n}\left(t, \xi_{0}, \eta_{0}\right)=q\left(t, \xi_{0}, n_{0}\right)<w(t)
$$

Using (2.21), we have that $\int_{t}^{\infty} q^{2}\left(s, \xi_{0}, n_{0}\right) d s<\int_{t}^{\infty} w^{2}(s) d s<\infty$ which contradicts the condition $\left(\mathrm{II}_{3}\right)$. This completes the proof. 
THEOREM 2.4. As sume that

$$
\int_{\alpha}^{\infty} \frac{d u}{\phi(u)}<\infty \text { and } \int_{-\alpha}^{-\infty} \frac{d u}{\phi(u)}<\infty, \quad \alpha>0
$$

Further assume that sequence $(2.18)$ for all positive constants $\xi$ and $\eta$ satisfies any one of the following conditions:

(III) there is a positive integer m such that $q_{n}(t, \xi, n)$ is defined for $\mathrm{n}=0,1,2 \ldots \mathrm{m}$, and

$$
\int_{t}^{\infty} q_{m}(s, \xi, n) d s=\infty
$$

$\left(\operatorname{III}_{2}\right) q_{n}(t, \xi, n)$ is defined for $n=0,1,2, \ldots$, and $\lim _{n \rightarrow \infty} q_{n}(t, \xi, n)=q(t, \xi, \eta)$ exists and satisfies

$$
\int_{t_{0}}^{\infty} q(s, \xi, n) d s=\infty
$$

Then Equation (1.1) is strongly os 111 atory.

PROOF. Assume that Equation $(1.4)$ at $\lambda=\lambda_{0}>0$, has a nonoscillatory solution $x(t)>0$ for $t>t_{0}$. The case $x(t)<0$ is handled similarly. Let $w(t)=\frac{x^{\prime}(t)}{\phi(x(h(t)))}$. By Lemmma 2.1 , we find that $(2.1)$ holds.

Suppose that (III $)$ holds, then, as proof of Theorem 2.3,

$$
q_{m}\left(t, \xi_{0}, \eta_{0}\right)=\xi_{0} \int_{t}^{\infty} q_{m}^{2}\left(s, \xi_{0}, \eta_{0}\right) d s+q_{0}\left(t, \xi_{0}, \eta_{0}\right)<w(t)
$$

or

$$
q_{m}\left(t, \xi_{0}, \eta_{0}\right)<\frac{x^{\prime}(t)}{\phi(x(h(t)))}<\frac{x^{\prime}\left(h(t) h^{\prime}(t)\right.}{k \phi(x(h(t)))} \text {. }
$$

From (2.22) and (2.23), we have that

$$
\lim _{t \rightarrow \infty} \int_{t_{0}}^{t} q_{m}\left(t, \xi_{0}, \eta_{0}\right) d s<\lim _{t \rightarrow \infty} \int_{x\left(h\left(t_{0}\right)\right)}^{x(h(t))} \frac{d u}{k \phi(u)}<\infty .
$$

This contradicts condition ( III $_{1}$ ).

Suppose that ( $\mathrm{III}_{2}$ ) holds, then it follows from (2.23) that

$\lim _{m \rightarrow \infty} q\left(t, \xi_{0}, \eta_{0}\right)<\frac{x^{\prime}\left(h(t) h^{\prime}(t)\right.}{k \phi(x(h(t)))}$, namely $q\left(t_{\delta} \xi_{0}, \eta_{0}\right)<\frac{x^{\prime}\left(h(t) h^{\prime}(t)\right.}{k \phi(x(h(t)))}$.

Hence

$$
\lim _{t \rightarrow \infty} \int_{t_{0}}^{t} q\left(s, \xi_{0}, n_{0}\right) d s<\lim _{t \rightarrow \infty} \int_{x\left(h\left(t_{0}\right)\right)}^{x(h(t))} \frac{d u}{\phi(u)}<\infty .
$$

which is again a contradiction, and the proof of the theorem is complete.

Equation ( 1.1 ) is said to be strongly bounded oscillatory if all bounded solutions of Equation (1.4) for any $\lambda \varepsilon(0, \infty)$ are oscillatory. 
Erom the prouf of Theorem (2.4), we see that the following result holds.

COROLLARY 2.5. As sune that the condition ( $I_{1}$ ) or ( $I_{1} I_{2}$ ) holds, then Equation (1.1) is strongly bounded oscillatory.

ACKNOWLEDGEMENT. The author would like to thank the referee for his valuable suggestions.

\section{REFERENCES}

1. BRADLEY, J.S. Oscillation theorems for a second order delay equation, J. Differential Equations, 8 (1970), 397-403.

2. CHIOU, K.L. Oscillation and nonoscillation theorems for second order functional differential equations, J. Math. Anal. Appl. 45 (1974), 382-403.

3. ERBE, L. Oscillation criteria for second order nonlinear delay equations, Canad. Math. Bul1. 16 (1973), 49-56.

4. GOLlWITZER, H.E. On nonlinear oscillations for a second order delay equation, J. Math. Anal. Appl. 26 (1969), 385-389.

5. LADAS, G. Oscillation and as ymptotic behavior of solutions of differential equations with retarded argunent, J. Differential Equations, 10 (1971), 281290 .

6. TRAVIS, C.C. Oscillation theorems for second-order differential equations with functional arguments, Proc. Aner. Math. Soc. 31 (1972), 199-202.

7. WALTMAN, A. A note on an oscillation criterion for an equation with a functional argument, Canad. Math. Bul1. 11 (1968), 593-595.

8. WONG, J.S.W. Second order oscillation with retarted arguments, in "Ordinary Differential Equations", Academic Press, New York/London, (1972), 581-596.

9. WINTNER, A. A criterion of oscillatory stability, Quart. Appl. Math. 7 (1949), $115-117$.

10. LEIGHTON, W. On self-adjoint differential equations of second order, J. London Math. Soc. 27 (1952), 37-47.

11. YAN, J. Oscillatory property of second order nonlinear differential equations with deviating argument Kexue Tongbao, 27 (1982), 7-11.

12. NEHARI, Z. Oscillation Criteria for second-order linear differential equations, Trans. Ame r. Math. Soc. 85 (1957), 428-445.

13. WINTNER, A On the non-existence of conjugate points, Amer. J. Math. 73 (1951), $368-380$. 


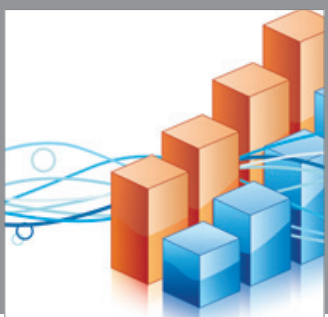

Advances in

Operations Research

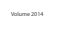

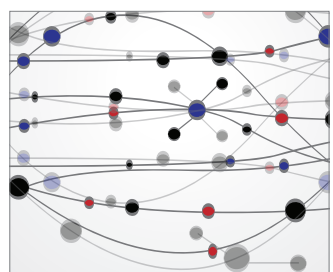

\section{The Scientific} World Journal
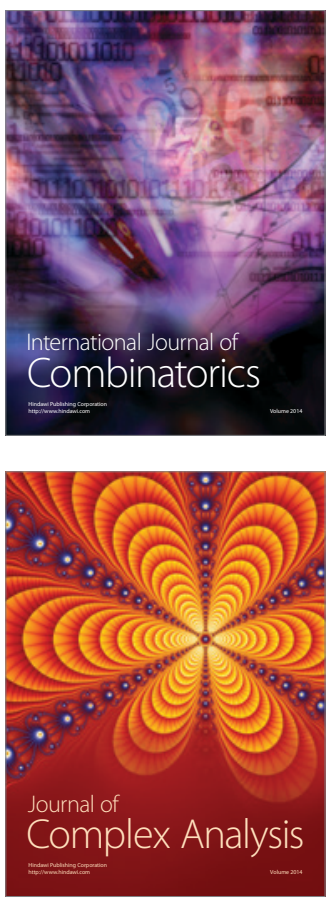

International Journal of

Mathematics and

Mathematical

Sciences
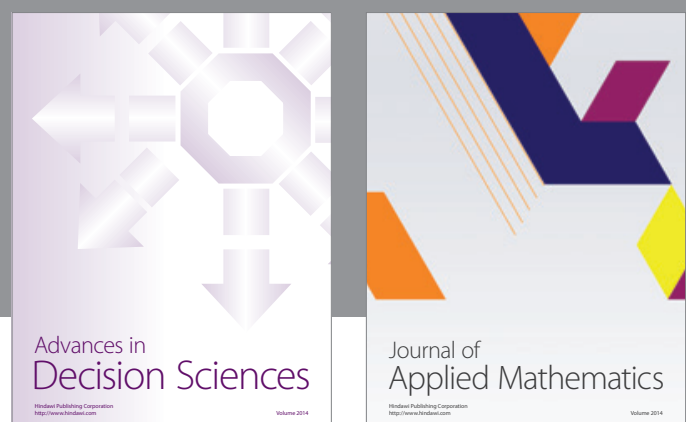

Journal of

Applied Mathematics
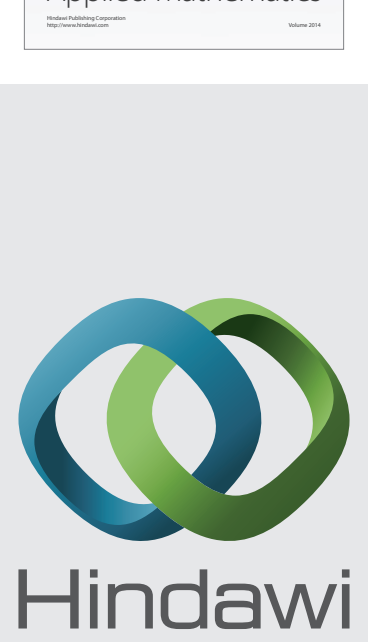

Submit your manuscripts at http://www.hindawi.com
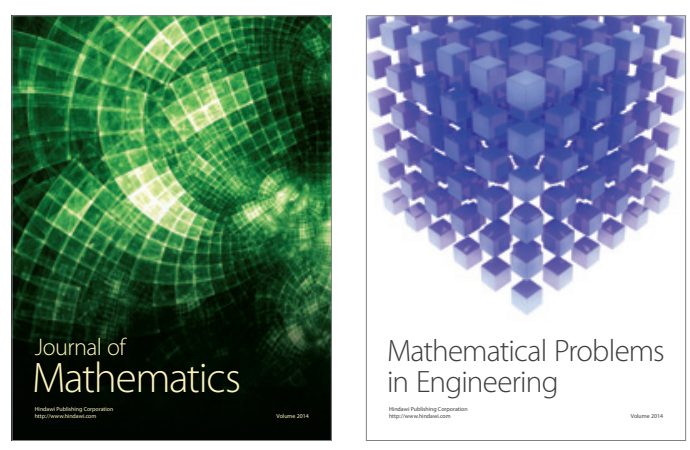

Mathematical Problems in Engineering
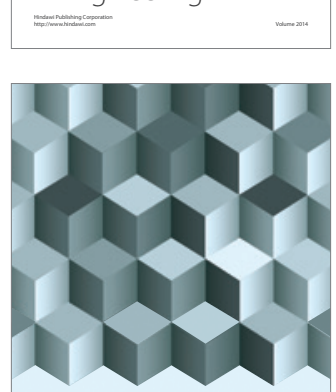

Journal of

Function Spaces
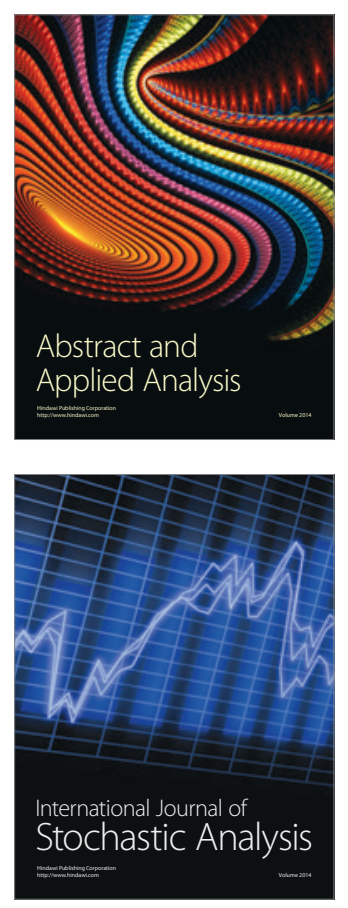

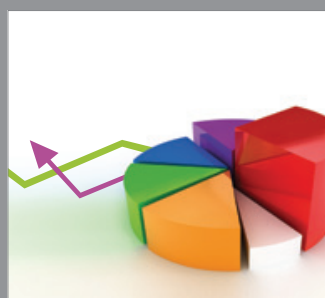

ournal of

Probability and Statistics

Promensencen
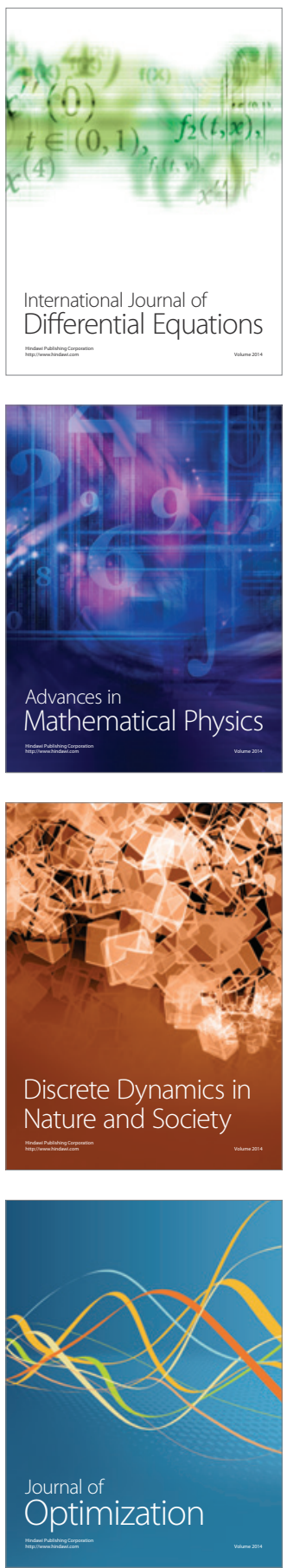